\title{
Multimodal imaging findings in normal-appearing white matter of leucoaraiosis: a review
}

To cite: Zhong G, Lou M. Multimodal imaging findings in normal-appearing white matter of leucoaraiosis: a review. Stroke and Vascular Neurology 2016;1:e000021. doi:10.1136/svn-2016000021

Received 7 April 2016 Revised 23 April 2016 Accepted 26 April 2016 Published Online First 19 May 2016

\section{CrossMark}

Department of Neurology, The Second Affiliated Hospital of Zhejiang University, School of Medicine, Hangzhou, Zhenjiang, China

Correspondence to Dr Min Lou; loumingxc@vip.sina.com

\section{ABSTRACT}

Leucoaraiosis (LA), also referred to as white matter hyperintensities (WMHs), are usually seen as patchy or confluent hyperintense areas on T2-weighted or fluid-attenuated inversion recovery MRI in the elderly. It is often asymptomatic in its early stages, yet its persistent evolution to more advanced stages may lead to substantial neurological dysfunction including dementia, stroke and death. Despite its clinical significance, the pathogenic mechanisms underlying LA development are uncertain. In patients with $L A$, the pathophysiological changes in white matter (WM) are suggested to be continuous from WMHs to its neighbourhood 'normal-appearing white matter (NAWM)' on conventional MRI sequences. Multimodal imaging studies revealed that the so-called 'NAWM' was actually abnormal with regard to underlying haemodynamic and microstructural changes. On the basis of positron emission tomography CT, xenon-CT, perfusion MRI, etc, the cerebral blood flow of NAWM was found to be significantly reduced in patients with $\mathrm{LA}$, compared with healthy controls. Meanwhile, the integrity of microstructures and blood-brain barrier in NAWM was also demonstrated to be impaired with diffusion tensor imaging and dynamic contrastenhanced MRI studies, respectively. In addition,the integrity of NAWM correlated much stronger with cognitive performance than did WMHs load. It is reasonable to assume that the subtle injury of NAWM would be more reversible than WMHs themselves. Therefore, multimodal imaging modalities could be appropriately applied to future interventional studies targeting at early pathophysiological changes of NAWM. In this paper, we summarise current knowledge about NAWM of LA mainly acquired from multimodal imaging studies in vivo, and attempt to give options for future work.

\section{INTRODUCTION}

Leucoaraiosis (LA), also referred to as white matter hyperintensities (WMHs), are usually seen as punctate, patchy or confluent hyperintense areas on T2-weighted or fluid-attenuated inversion recovery (FLAIR) MRI scans in the elderly population. The prevalence of LA is $>80 \%$ between 60 and 70 years, and approaching $100 \%$ between 80 and 90 years. ${ }^{1}$ LA is often asymptomatic in its early stages, yet its evolution to more advanced stages may lead to substantial neurological dysfunction including dementia, depression, gait disturbance, stroke and death. ${ }^{23}$ Despite its clinical significance, the pathogenic mechanisms underlying LA have not been fully elucidated.

The alterations of white matter (WM) architecture in LA are suggested to be continuous from WMHs to its neighbourhood 'normal-appearing white matter (NAWM)' on conventional MRI sequences. ${ }^{4}$ Clinically, the disruption of NAWM integrity reflected by diffusion tensor imaging (DTI) parameters correlated stronger with psychomotor dysfunction than did WMHs load. ${ }^{5}$ Accumulating evidences advocate the idea that WMHs may represent merely a 'tip of the iceberg' of the true extent of pathophysiological changes underlying global WM. In virtue of imaging technological innovation, multimodal imaging modalities have been applied to explore the haemodynamic and microstructural changes of NAWM during the past decades. Undoubtedly, these efforts could deepen our understanding about the intrinsic biological mechanisms underlying the dynamic evolution process of WMHs, which is prerequisite for adopting effective measures to prevent or retard progression of LA in its infant stages. In this review, we thus summarise current knowledge about NAWM of LA mainly acquired from multimodal imaging studies, and attempt to give options for future work.

Haemodynamic disturbance in NAWM and its relationship with progression of LA

Chronic hypoperfusion secondary to arteriosclerosis of small perforating arteries has long been proposed as the pathogenesis of LA. Supporting this assumption, haemodynamic studies have shown that perfusion reserve, cerebrovascular reactivity and dynamic autoregulation function were compromised in 
patients with LA. ${ }^{6}$ Direct evidence of reduced perfusion in WM of LA has been acquired from studies using positron emission tomography (PET), single-photon emission CT (SPECT) and xenon-CT. However, it is uncertain whether hypoperfusion is a primary pathogenic mechanism or simply a secondary response to downregulation of cerebral blood flow (CBF) for reduced metabolic demand in patients with LA. Researchers assumed that hypoperfusion might already exist in NAWM preceding its evolution to visible WMHs on T2-weighted or FLAIR images; in case of WMHs, expansion was primarily due to chronic ischaemia.

In 2002, O'Sullivan et al first demonstrated that CBF of periventricular NAWM in patients with ischaemic LA was significantly reduced, compared with age-matched controls, using quantitative perfusion MRI. When the analysis was confined to patients with hypertension, both $\mathrm{CBF}$ of periventricular and centrum semiovale NAWM were obviously reduced. In addition, they found that CBF was lower in periventricular NAWM than that in centrum semiovale NAWM both in patients and controls, which was consistent with the phenomenon that WMHs tends to emerge earlier in periventricular WM than in centrum semiovale. Subsequent studies further demonstrated that the degree of CBF reduction in NAWM was associated with the severity of LA. ${ }^{9}$

Recently, a prospective study revealed that a low baseline CBF of NAWM was an independent predictor of subsequent development of WMHs in patients with minor stroke/transient ischaemic attack (TIA) after 18-month observation, and the odds of having new WMHs was reduced by 0.61 for each increase of $1 \mathrm{~mL} /$ $100 \mathrm{~g} / \mathrm{min}$ with baseline CBF of NAWM. ${ }^{10}$ Similarly, another contemporary study found that NAWM transformed into new WMH at follow-up had a significantly lower baseline CBF than that of the remaining NAWM. ${ }^{11}$ Furthermore, mean CBF in NAWM surrounding the index WMHs was lower than that in the total NAWM, extending $\sim 12 \mathrm{~mm}$ from the edge of WMHs. This is consistent with the pattern of LA progression, mainly expanding from the edge of index WMHs other than those emerging in more distal NAWM. Nevertheless, a large sample study, which enrolled 575 patients with manifest arterial diseases, failed to establish the association between global baseline $\mathrm{CBF}$ and progression of WMHs after 3.9 years follow-up. ${ }^{12}$ The main distinction is that this study quantified mean CBF of the whole brain other than CBF of NAWM as used in other two studies. Actually, a recent study confirmed that the association of WMHs severity with intracranial atherosclerotic stenosis (ICAS) was stronger than that with extracranial atherosclerotic stenosis (ECAS) ${ }^{13}$ The hemodynamic insufficiency in periventricular border zone would occur more easily in ICAS than ECAS. Taking these findings together, it is thus conceivable to infer that progression of WMHs may be mainly driven by regional rather than global hypoperfusion.

\section{Disruption of microstructures in NAWM}

Substantial evidences have linked LA with cognitive dysfunction in the elderly, particularly in domains of information processing speed and executive function. ${ }^{2}$ However, the association between the burden of LA and severity of cognitive impairment was weak. One explanation is that the underlying subtle microstructural changes in NAWM may also contribute to cognitive impairment, which was supported by the findings from diffusion weighted imaging (DWI) and DTI studies. ${ }^{5}$

\section{DWI findings in NAWM}

DWI could reflect the freedom of water molecules movement, which is restricted by myelin, axon and other cell structures. Any disruption to these structures may lead to elevated apparent diffusion coefficient (ADC). The $\mathrm{ADC}$ values of the whole brain or WMHs were found to be positively correlated with LA extension. ${ }^{14}$ Combining DWI with MR spectroscopy (MRS), Firbank $e t a l^{15}$ found that ADC as well as the metabolite ratios of $\mathrm{N}$-acetylaspartate/creatine and N-acetylaspartate/ choline of NAWM was significantly correlated with total WMHs volume. MRS could quantify the levels of N-acetylaspartate (NAA), which would be decreased due to the damage of neurons and their associated axons. On the basis of ADC histogram analysis, the peak height of the ADC histogram of normal-appearing brain tissue (NABT) was independently associated with multiple cognitive domains of patients with LA after adjusting for WMHs volume and brain atrophy. ${ }^{16}$ A prospective study also demonstrated that baseline ADC metrics of NABT outperformed the same metrics of WMHs lesions in predicting deterioration of cognitive and functional outcomes after 3-year follow-up. ${ }^{17}$

\section{DTI findings in NAWM}

DTI can non-invasively provide quantitative information about disruption of microscopic architecture underlying WM of LA. DTI measures the diffusion in at least six non-collinear directions, providing a three-dimensional representation of water motion. The most commonly used parameters are fractional anisotropy (FA) and mean diffusivity (MD). FA reflects the directionality of diffusion, while MD is a measure of diffusion averaged in all spatial directions. DTI has been extensively used to characterise the subtle pathological changes underlying NAWM, which may be invisible on conventional MRI sequences. In 1999, Derek et al found that FA of periventricular WM was significantly decreased in ischaemic LA compared with normal controls, while its MD was increased. ${ }^{18}$ However, this small study lacked the spatial resolution to establish whether these changes were confined to WMHs or involved with NAWM. Subsequent studies further demonstrated increased $\mathrm{MD}$ and decreased FA in NAWM of patients with LA compared with healthy controls, ${ }^{19}$ and the changes of FA and MD were quantitatively associated with the severity of WMHs. ${ }^{20}$ 
Maillard $e t a t^{21}$ found that FA in different regions of NAWM was inversely correlated with the number and proximity of WMHs in its vicinity, suggesting a 'penumbra' surrounding index WMHs (as illustrated in figure 1). The WM integrity of the penumbra may be more subtly disrupted and expected to be more vulnerable than other NAWM. Consistent with this assumption, prospective studies showed that $80 \%$ of new WMHs had been emerging as direct extensions of pre-existing lesions other than isolated new lesions, ${ }^{22}$ and lower baseline FA in NAWM independently predicted greater risk of transition to WMHs. ${ }^{23}$ However, Maillard et al recently demonstrated that the losses of WM integrity over time in the 'WMHs penumbra' were similar in magnitude to other NAWM as well as WMHs themselves. This finding suggested that different regions of WM lie along a continuum of injury in patients with LA, and the 'WMHs penumbra' appeared to have no distinctive temporal course of deterioration. Whether the conception of the 'WMHs penumbra' is of clinical significance has to be tested in future interventional studies.

\section{Disruption of the blood-brain barrier (BBB) in NAWM}

BBB failure and endothelial dysfunction are also proposed to be important pathophysiological mechanisms for WMHs development. Increased permeability of BBB may give rise to leakage of serum components into and through the walls of cerebral small vessels leading to lipohyalinosis, perivascular oedema, demyelination, loss of neurons and gliosis. ${ }^{24}$

In the early dates, the permeability of BBB was mainly assessed on the basis of the cerebrospinal fluid (CSF)/ serum ratio for albumin, which was expected to be elevated in proportion to the severity of endothelium damage of cerebral small vessels. Obviously, this method is not suitable for screening or dynamic monitoring of BBB permeability in general populations for its invasive and non-localisation properties. Dynamic contrast-enhanced MRI (DCE-MRI) is a new method being applied to assess subtle BBB disruption in humans, and with considerable

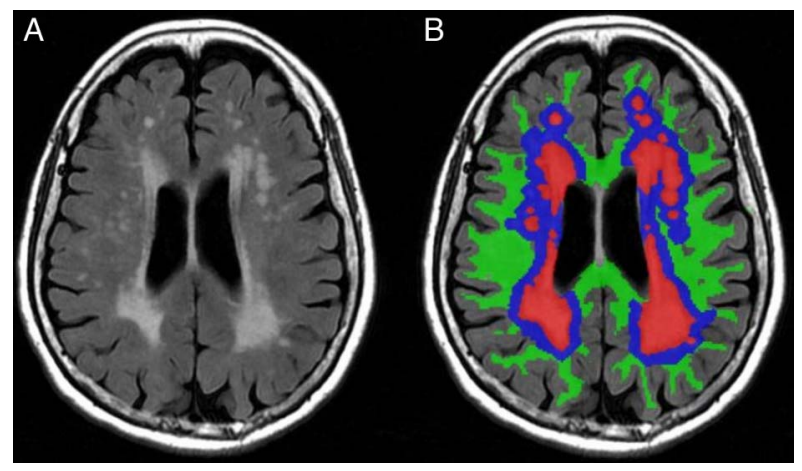

Figure 1 (A) Axial T2-weighted fluid-attenuated inversion recovery image; $(B)$ coloured version of image $(A)$ : white matter hyperintensities (WMHs; red areas); penumbra surrounding index WMHs in normal-appearing white matter (NAWM; blue areas); other regions of NAWM (green areas). reliability. On the basis of DCE-MRI, signal changes consistent with increased BBB permeability were detected in NAWM and WMHs in patients with LA compared with healthy controls. ${ }^{25}{ }^{26}$ Recently, a study among patients with Binswanger disease demonstrated that abnormal WM permeability (WMP) was more frequently detected in NAWM than in WMHs, with the highest frequency in a $4 \mathrm{~mm}$ width ring surrounding the WMHs, which coincided with the aforementioned finding that the surrounding NAWM was vulnerable, leading to progression of WMHs. ${ }^{27}$

\section{Histopathological and postmortem imaging findings in NAWM}

Autopsy studies confirmed that the underlying pathological changes of LA extended into the NAWM. Among six $\mathrm{AD}$ patients with LA, neuropathological examination revealed much more extensive changes in WM than did parallel postmortem MRI, with a mean of $54 \%$ larger abnormal areas. ${ }^{28}$ The pathological lesions not identified on T2-weighted MRI represented minor changes with a lower intensity of myelin staining. A histopathological study also demonstrated that afferent microvascular density was significantly lower both in deep WMHs lesions and NAWM of patients with LA than in those of healthy subjects. ${ }^{29}$ These differences were most prominent in patients who died before 60, indicating that global microvascular rarefaction in LA is not completely age-dependent. Interpretation of these results should be done cautiously, as they usually represent a late-stage condition of tissue damage rather than the initial pathological alterations of LA.

\section{Current evaluation of LA progression and its limitations}

Progression of LA has been linked to cognitive worsening, ${ }^{30}$ increased risk of depression ${ }^{3}$ and mortality. ${ }^{31}$ The reported prevalence and speed of LA progression differ greatly in various cohorts due to the different duration of observation and definition of progression. In the Austrian Stroke Prevention Study (ASPS) with healthy volunteers aged $50-75$ years, participants with early confluent and confluent WMHs at baseline underwent median increases of 2.7 (IQR $0.5-5.9$ ) $\mathrm{cm}^{3}$ and 9.3(7.121.0) $\mathrm{cm}^{3}$, respectively, in lesion volume over 6 years. ${ }^{32}$ In contrast, the change in WMHs volume was negligible in their counterparts without WMHs or with punctate lesions at baseline. The hospital-based multicentre, multinational Leukoaraiosis and Disability (LADIS) study showed that $\sim 74 \%$ of participants experienced assessable WMHs progression after 3-year follow-up, especially in the frontal and parietal lobes. ${ }^{33}$

Hitherto, progression of LA was generally assessed on the basis of changes of WMHs volume or severity grade. Long-term follow-up (usually $\geq 3$-year) was needed to observe such an appreciable change because the evolution process of LA is pretty slow. Up to $43 \%$ of participants were reportedly lost to follow-up after 3-year observation. ${ }^{34}$ Those who completed the follow-up examinations were usually younger and had less vascular 
risk factors than did the whole study population, leading to an underestimation of LA progression risk. When observed thus, it is particularly not feasible to evaluate the effect of certain therapeutic interventions aiming at retardation of LA progression.

\section{Clinical implications of multimodal imaging studies in NAWIM}

Promisingly, multimodal imaging modalities targeting NAWM could help us to detect and monitor subtle pathophysiological changes in early stages of LA formation. Small studies have shown that CBF could be increased by the use of sympathomimetic midodrine ${ }^{35}$ or angiotensin receptor blockers, ${ }^{36}$ as well as fitness exercises. ${ }^{37}$ However, whether these possible interventions could be applied to prevent LA progression needs further investigation in clinical trials. In the future, the use of non-invasive arterial spin-labelling (ASL) could help to find out whether progression of LA will be accelerated when cerebral perfusion declines to a certain threshold, since it could monitor the dynamic changes of CBF in WM.

Preliminary studies have shown that the changes of FA and MD in NAWM were detectable over a 1 year follow-up. ${ }^{38}$ In contrast, over this period, there was no change in conventional MRI parameters, including brain volume, WMHs lesion load and lacunar infarct number, as well as changes of cognitive metrics. Recently, another study conducted among community-dwelling people of similar age suggested that MD may provide the best sensitivity in discrimination between WMHs and NAWM even with a mild WMHs load, among other parameters of FA, magnetisation transfer ratio (MTR) and longitudinal relaxation time (T1). The reduction of MTR indicates changes in the underlying composition of tissue, especially the abnormalities of myelin. Moreover, substantial evidence demonstrated that the integrity of microstructure in NAWM, represented by DTI parameters, correlated much closer with cognitive performance than did the load of WMHs, lacunar infarcts or brain volume in patients with LA. ${ }^{5}$ Thus, use of DTI parameters as outcome variables would be expected to more efficiently evaluate the efficacy of therapeutic interventions designed for delaying LA progression than the simple measurement of WMHs volume or cognitive deterioration in clinical trials. To be mentioned here, there is another emerging technique, known as 'diffusion kurtosis imaging (DKI)', which might be even more sensitive than DTI to detect subtle microstructural changes in NAWM of LA in the near future, as indicated in relevant studies of patients with multiple sclerosis (MS). ${ }^{39}$

A dynamic monitor of BBB permeability may also be used to evaluate the efficacy of medications aiming at endothelium or BBB protection. For example, vitamin B supplementation was found to effectively delay WMHs progression for patients with recent stroke and severe cerebral small vessel disease. ${ }^{40}$ Currently, the proposed mechanism is that vitamin B may protect the endothelium by reducing concentrations of homocysteine. Future studies using DCE-MRI may help to confirm it by measurement of BBB permeability.

\section{CONCLUSIONS}

The clinical significance of LA has obtained increasing attention with the arrival of an ageing society. LA develops in an insidious way, while progressing persistently. The so-called 'NAWM' has revealed early pathophysiological abnormalities. The pathogenic role of chronic ischaemia in the development of LA was further corroborated with the findings that hypoperfusion exists in NAWM prior to its transition to WMHs. Multimodal imaging modalities could thus be appropriately applied to detect and monitor subtle microstructural and haemodynamic changes in NAWM, in terms of future interventional studies. Moreover, it is reasonable to assume that the pathophysiological changes in NAWM would be more reversible than WMHs itself, since NAWM occupies the main body of global WM and any damage to the integrity of the WM network may exert a negative impact on brain function. Therefore, preventive and therapeutic strategies targeting early changes in NAWM would be expected to be more promising and rewarding.

Contributors GZ conceived and drafted the review with guidance from ML, who made a critical revision of the manuscript.

Funding This work was supported and funded by grants from the Science Technology Department of Zhejiang Province (2013C03043-3) and the National Natural Science Foundation of China (NSFC) (81471170).

Competing interests None declared.

Provenance and peer review Commissioned; externally peer reviewed.

Data sharing statement No additional data are available.

Open Access This is an Open Access article distributed in accordance with the Creative Commons Attribution Non Commercial (CC BY-NC 4.0) license, which permits others to distribute, remix, adapt, build upon this work noncommercially, and license their derivative works on different terms, provided the original work is properly cited and the use is non-commercial. See: http:// creativecommons.org/licenses/by-nc/4.0/

\section{REFERENCES}

1. de Leeuw FE, de Groot JC, Achten E, et al. Prevalence of cerebral white matter lesions in elderly people: a population based magnetic resonance imaging study. The Rotterdam Scan Study. J Neurol Neurosurg Psychiatry 2001;70:9-14.

2. Debette S, Markus HS. The clinical importance of white matter hyperintensities on brain magnetic resonance imaging: systematic review and meta-analysis. BMJ 2010;341:c3666.

3. Firbank MJ, Teodorczuk A, van der Flier WM, et al. Relationship between progression of brain white matter changes and late-life depression: 3-year results from the LADIS study. Br J Psychiatry 2012;201:40-5.

4. Maillard P, Fletcher E, Lockhart SN, et al. White matter hyperintensities and their penumbra lie along a continuum of injury in the aging brain. Stroke 2014;45:1721-6.

5. Vernooij MW, Ikram MA, Vrooman HA, et al. White matter microstructural integrity and cognitive function in a general elderly population. Arch Gen Psychiatry 2009;66:545-53.

6. Tomura N, Sasaki K, Kidani H, et al. Reduced perfusion reserve in leukoaraiosis demonstrated using acetazolamide challenge 123I-IMP. J Comput Assist Tomogr 2007;31:884-7. 
7. Purkayastha S, Fadar O, Mehregan A, et al. Impaired cerebrovascular hemodynamics are associated with cerebral white matter damage. J Cereb Blood Flow Metab 2014;34:228-34.

8. O'Sullivan M, Lythgoe DJ, Pereira AC, et al. Patterns of cerebral blood flow reduction in patients with ischemic leukoaraiosis. Neurology 2002;59:321-6.

9. Fu J, Tang J, Han J, et al. The reduction of regional cerebral blood flow in normal-appearing white matter is associated with the severity of white matter lesions in elderly: a Xeon-CT study. PLoS One 2014;9:e112832.

10. Bernbaum M, Menon BK, Fick G, et al. Reduced blood flow in normal white matter predicts development of leukoaraiosis. J Cereb Blood Flow Metab 2015;35:1610-15.

11. Promjunyakul N, Lahna D, Kaye JA, et al. Characterizing the white matter hyperintensity penumbra with cerebral blood flow measures. Neuroimage Clin 2015;8:224-9.

12. van der Veen $\mathrm{PH}$, Muller M, Vincken $\mathrm{KL}$, et al. Longitudinal relationship between cerebral small-vessel disease and cerebral blood flow: the second manifestations of arterial disease-magnetic resonance study. Stroke 2015;46:1233-8.

13. Park JH, Kwon HM, Lee J, et al. Association of intracranial atherosclerotic stenosis with severity of white matter hyperintensities. Eur J Neurol 2015;22:44-52.

14. Mascalchi M, Tessa $\mathrm{C}$, Moretti $\mathrm{M}$, et al. Whole brain apparent diffusion coefficient histogram: a new tool for evaluation of leukoaraiosis. J Magn Reson Imaging 2002;15:144-8.

15. Firbank MJ, Minett T, O'Brien JT. Changes in DWI and MRS associated with white matter hyperintensities in elderly patients. Neurology 2003;61:950-4.

16. Schmidt R, Ropele S, Ferro J, et al. Diffusion-weighted imaging and cognition in the leukoaraiosis and disability in the elderly study. Stroke 2010;41:e402-8.

17. Jokinen $H$, Schmidt $R$, Ropele $S$, et al. Diffusion changes predict cognitive and functional outcome: the LADIS study. Ann Neurol 2013;73:576-83.

18. Jones DK, Lythgoe D, Horsfield MA, et al. Characterization of white matter damage in ischemic leukoaraiosis with diffusion tensor MRI. Stroke 1999;30:393-7.

19. O'Sullivan M, Summers PE, Jones DK, et al. Normal-appearing white matter in ischemic leukoaraiosis: a diffusion tensor MRI study. Neurology 2001;57:2307-10.

20. Maniega SM, Valdés Hernández MC, Clayden JD, et al. White matter hyperintensities and normal-appearing white matter integrity in the aging brain. Neurobiol Aging 2015;36:909-18.

21. Maillard P, Fletcher E, Harvey D, et al. White matter hyperintensity penumbra. Stroke 2011;42:1917-22.

22. Maillard $\mathrm{P}$, Carmichael $\mathrm{O}$, Fletcher $\mathrm{E}$, et al. Coevolution of white matter hyperintensities and cognition in the elderly. Neurology 2012;79:442-8.

23. de Groot M, Verhaaren BF, de Boer R, et al. Changes in normal-appearing white matter precede development of white matter lesions. Stroke 2013;44:1037-42.

24. Wardlaw JM, Sandercock PA, Dennis MS, et al. Is breakdown of the blood-brain barrier responsible for lacunar stroke, leukoaraiosis, and dementia? Stroke 2003;34:806-12.
25. Hanyu H, Asano T, Tanaka $\mathrm{Y}$, et al. Increased blood-brain barrier permeability in white matter lesions of Binswanger's disease evaluated by contrast-enhanced MRI. Dement Geriatr Cogn Disord 2002;14:1-6.

26. Topakian R, Barrick TR, Howe FA, et al. Blood-brain barrier permeability is increased in normal-appearing white matter in patients with lacunar stroke and leucoaraiosis. J Neurol Neurosurg Psychiatr 2010;81:192-7.

27. Huisa BN, Caprihan A, Thompson J, et al. Long-term blood-brain barrier permeability changes in Binswanger disease. Stroke 2015;46:2413-18

28. Bronge L, Bogdanovic N, Wahlund LO. Postmortem MRI and histopathology of white matter changes in Alzheimer brains. A quantitative, comparative study. Dement Geriatr Cogn Disord 2002;13:205-12.

29. Moody DM, Thore CR, Anstrom JA, et al. Quantification of afferent vessels shows reduced brain vascular density in subjects with leukoaraiosis. Radiology 2004;233:883-90.

30. Kloppenborg RP, Nederkoorn PJ, Geerlings MI, et al. Presence and progression of White matter hyperintensities and cognition: a meta-analysis. Neurology 2014;82:2127-38.

31. Sabayan B, van der Grond J, Westendorp RG, et al. Accelerated progression of white matter hyperintensities and subsequent risk of mortality: a 12-year follow-up study. Neurobiol Aging 2015;36:2130-5.

32. Schmidt R, Enzinger C, Ropele S, et al. Progression of cerebral white matter lesions: 6-year results of the Austrian Stroke Prevention Study. Lancet 2003;361:2046-8.

33. Gouw AA, van der Flier WM, Fazekas F, et al. Progression of white matter hyperintensities and incidence of new lacunes over a 3-year period: the Leukoaraiosis and Disability study. Stroke 2008;39:1414-20.

34. Schmidt R, Ropele S, Enzinger C, et al. White matter lesion progression, brain atrophy, and cognitive decline: the Austrian stroke prevention study. Ann Neurol 2005;58:610-16.

35. Duschek S, Hadjamu M, Schandry R. Enhancement of cerebral blood flow and cognitive performance following pharmacological blood pressure elevation in chronic hypotension. Psychophysiology 2007;4:145-53.

36. Hajiar I, Rodgers K. Do angiotensin receptor blockers prevent Alzheimer's disease? Curr Opin Cardiol 2013;28:417-25.

37. Ainslie PN, Cotter JD, George KP, et al. Elevation in cerebral blood flow velocity with aerobic fitness throughout healthy human ageing. J Physiol (Lond) 2008;586:4005-10.

38. Nitkunan A, Barrick TR, Charlton RA, et al. Multimodal MRI in cerebral small vessel disease: its relationship with cognition and sensitivity to change over time. Stroke 2008;39:1999-2005

39. Yoshida M, Hori M, Yokoyama K, et al. Diffusional kurtosis imaging of normal-appearing white matter in multiple sclerosis: preliminary clinical experience. Jpn J Radiol 2013:31:50-5.

40. Cavalieri M, Schmidt R, Chen $\mathrm{C}$, et al. B vitamins and magnetic resonance imaging-detected ischemic brain lesions in patients with recent transient ischemic attack or stroke: the VITAmins TO Prevent Stroke (VITATOPS) MRI-substudy. Stroke 2012:43:3266-70. 